\title{
Neonatal seizures associated with cerebral lesions shown by magnetic resonance imaging
}

\author{
H Leth, P B Toft, M Herning, B Peitersen, H C Lou
}

\begin{abstract}
Aim-To determine the diagnostic potential of magnetic resonance imaging (MRI) in neonatal seizures; to elucidate the aetiology, timing, and prognosis of the cerebral lesions detected.

Methods-Thirty one term neonates with clinical seizures underwent ultrasonography between days 1-7 (mean 2.5 days) and a high field spin-echo MRI scan on days 1-30 (mean 8.1 days), both of which were repeated at 3 months of age. Routine investigation excluded, as far as possible, infection, haematological, and metabolictoxic causes as causes of the neonatal seizures.

Results-Brain abnormality was demonstrated by MRI in $68 \%$ of infants and ultrasonographically in $10 \%$. Diffuse brain lesions (present in $29 \%$ ) were associated with high mortality (58\%) and morbidity $(42 \%)$, whatever the aetiology. In contrast to a better short term prognosis for neonates with focal lesions where no infants died, $33 \%$ had a handicap, and the rest were normal at a mean follow up age of $2 \frac{1}{2}$ years. Cerebral lesions were presumed to have antepartum origin in $43 \%$ of cases. Seizure aetiology was considered to be hypoxic-ischaemic in $35 \%$, haemorrhagic in $26 \%$, metabolic disturbances and cerebral dysgenesis in $16 \%$ and unknown in $23 \%$.
\end{abstract}

Conclusions-MRI detected a remarkably high incidence of brain lesions in neonatal seizures. Almost half of these were of prenatal origin and pathogenesis may essentially be attributed to hypoxic and/or haemodynamic causes.

(Arch Dis Child 1997;77:F105-F110)

The Danish Research Center for Magnetic Resonance

H Leth

M Herning

P B Toft

Department of Paediatrics,

University Hospital

Hvidovre

H Leth

B Peitersen

John F Kennedy Institute, Glostrup,

Denmark

H C Lou

Correspondence to:

Dr Helle Leth, Department

of Paediatrics, University

Hospital, Kettegaard Allé 30 2650 Hvidovre, Denmark.

Accepted 15 April 1997

Keywords: MRI; ultrasonography; seizures; cerebral lesions

Among the abnormal neurological signs in neonates, seizures have a particularly poor prognosis. ${ }^{1-5}$ Seizure activity usually implies an underlying neurological disorder with a wide spectrum of different aetiologies of which hypoxic-ischaemic encephalopathy is the most serious. ${ }^{5}$ Prognosis is therefore primarily determined by the nature, location, and extent of the underlying neuropathological process, which makes accurate description of the lesion essential.

Experience with magnetic resonance imaging (MRI) in demonstrating different neuropathology in the newborn is limited, but MRI seems to be more sensitive than computed tomography for the detection of subtle brain injuries. ${ }^{6-11}$ Recent MRI reports have suggested various patterns and timing of brain lesions in infants with hypoxic ischaemia. ${ }^{612-15}$

This study aimed prospectively to evaluate the role of MRI in describing the brain lesions associated with neonatal seizures and to correlate the lesions with clinical data to suggest the aetiology and possible timing of the brain lesions. A recent study of the role of MRI in identifying neonates with seizures at risk of very poor outcome found no correlation between MRI findings and presumed causes of perinatal cerebral injury. ${ }^{11}$

\section{Methods}

Thirty one term neonates (eight girls, 23 boys), with birthweights ranging from $2160-4780 \mathrm{~g}$ (mean $3223 \mathrm{~g}$ ), were studied. They had been admitted to the neonatal intensive care unit between June 1992 and March 1995 for seizures. They underwent MRI as soon as their clinical status and access to facilities permitted, at 1 to 30 days of life (mean 8.1 days), and again at 3 months of age. All inborn full term neonates presenting with clinical seizures during the research period were included. Four neonates were referred from other hospitals and their first scans were performed somewhat later (mean 20.2 days). As far as possible, routine investigation excluded infection, haematological, and metabolic-toxic factors as possible causes.

Three neonates required assisted ventilation during the imaging procedure. Apart from these infants, sedation was not necessary at the early scan. At 3 months of age, all infants were sedated with $75-150 \mathrm{mg} / \mathrm{kg}$ chloral hydrate rectally.
Neonatal seizures were diagnosed clinically and classified according to Volpe as: (1) subtle; (2) clonic; (3) tonic; and (4) myoclonic. ${ }^{16}$

Infants diagnosed as having neonatal encephalopathy were graded by clinical severity according to Sarnat. ${ }^{17}$ Hypoxic-ischaemic encephalopathy was diagnosed in infants who had three of the following five features: (1) bradycardia (heart rate $<80 /$ minute) or late decelerations on cardiotocography, or meconium stained amniotic fluid; (2) umbilical $\mathrm{pH}$ $<7.10$; (3) Apgar score of $<5$ at 5 minutes; (4) delayed onset of spontaneous respiration; (5) multiorgan failure.

An electroencephalogram was performed at a range of 1-8 days (mean 3.6 days) after the onset of clinical seizures, using a conventional 18 channel Sanei machine with an electrode 
Table 1 MRI patterns ( $T_{2}$-weighted images) in neonatal brain following perinatal asphyxia, according to Baenziger et $a l^{14}$

Pattern A: Diffuse hyperintensity of cerebral hemispheres Pattern B: Hyperintensity of parasagittal watershed regions Pattern C: Lesions in basal ganglia or thalamus

Pattern D: Periventricular hyperintensity

Pattern E: Focal parenchymal ischemia or haemorrhage

position corresponding to a $10 / 20$ system. Recording time was at least 30 minutes. The interictal EEG was classified as normal, unifocal, multifocal, periodic or flat.

All infants were accompanied by a neonatologist during MRI and monitored with pulse oximetry (Microspan 1040A, Scan-med A/S, Norway).
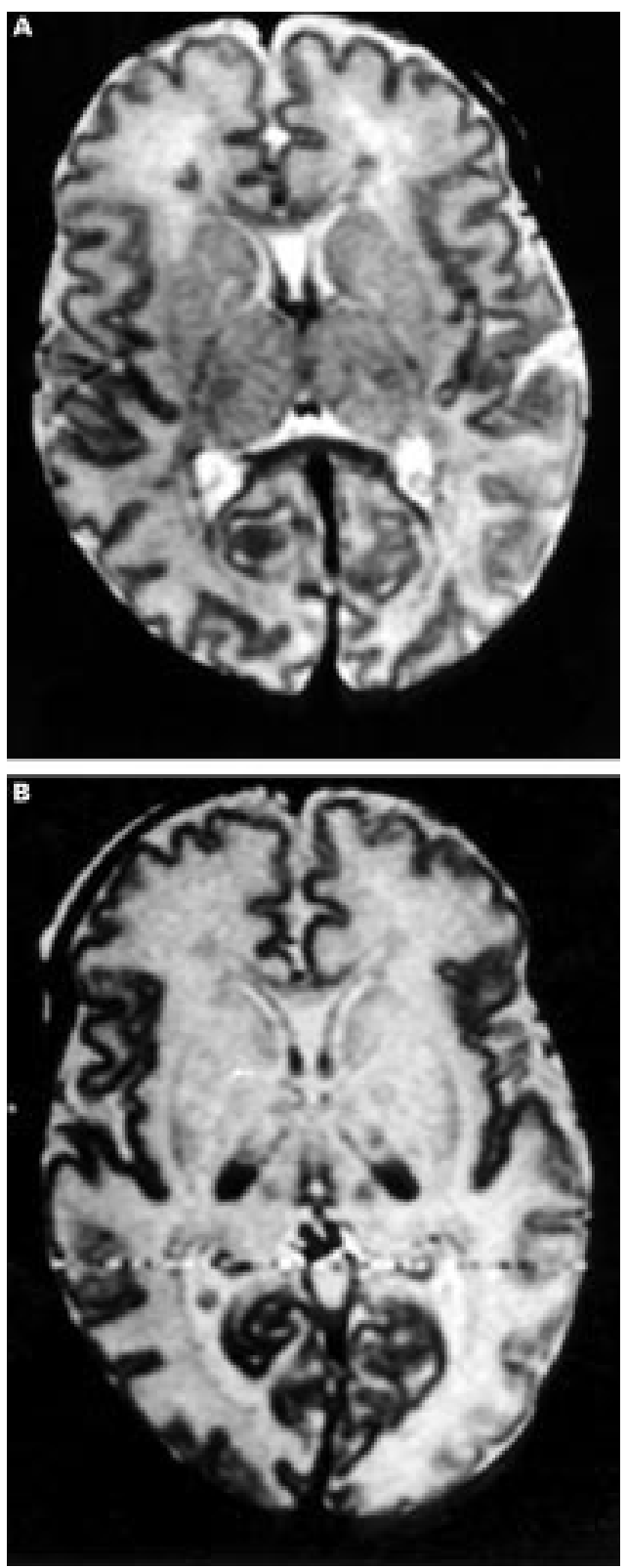

Figure 1 Case 2, a severely asphyxiated neonate, examined at 53 hours. Axial T2 weighted spin echo 3000/120 image at the level of basal ganglia showing diverse signal intensity from frontal and occipital white matter. An MRI scan five days later showed isointense central nuclei within the white matter due to widespread cellular necrosis.

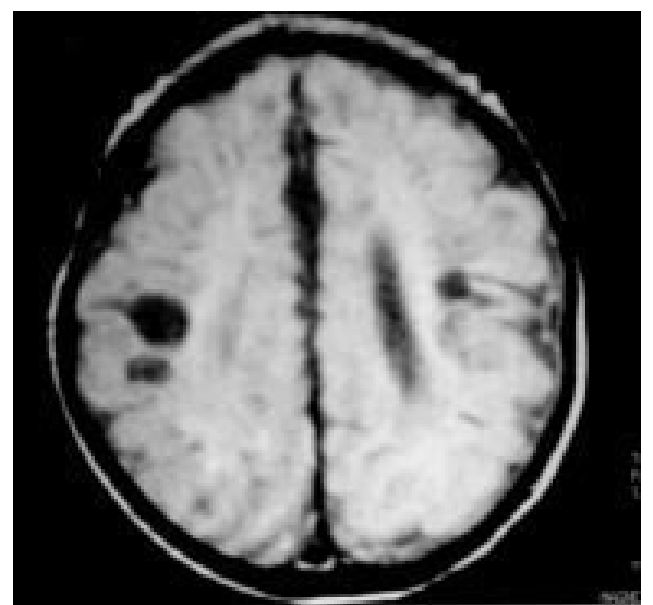

Figure 2 Proton density weighted coronal image 600/15 performed on the fourth day of life in case 9, showing low intensity subcortical cystic areas at least two to three weeks old (arrows). Increase in extracerebral space is also seen as a result of generalised cortical atrophy. Metabolic screening revealed molybdenum cofactor deficiency.

The study was approved by the local ethics committee and parental consent was obtained for each infant.

Neurological examination was performed at discharge, 5 months, and 1 and 2 years of age by two neonatologists, one of whom was blinded to the MRI findings.

NEUROIMAGING

Magnetic resonance imaging was performed on a 1.5 Siemens SP 63/84 scanner and as a minimum included axial $4 \mathrm{~mm} \mathrm{~T} 1$ and T2 weighted spin-echo images, 550/14 (TR/TE) and $3000 / 22,60,120$ and sagittal T1 weighted images, using a $256^{\star} 256$ imaging matrix.

The nature and topography of brain injury varies with the severity of the injury and the age of the patient at the time of injury. ${ }^{7-9} 1518-20$

Acute ischaemic lesions were assessed from deviations in the signal intensities in the newborn brain. Basal ganglia, brain stem, and cortex are normally hypointense relative to the cerebral white matter, which is normally homogeneous and hyperintense in T2 images. The early ischaemic lesions were graded, based on T2 weighted images, according to Baenziger et $a{ }^{14}$ (table 1) All MRIs were assessed blind.

MRI scans obtained within the first two to three days after asphyxia will show low signal (dark areas) on $\mathrm{T} 1$ weighted images and high signal (bright areas) on T2 weighted images in the affected regions. This is followed by gradual $\mathrm{T} 1$ and $\mathrm{T} 2$ shortening (high signal on $\mathrm{T} 1$ and low signal on T2 weighted images) from days 4-5 during the first months. ${ }^{15}$

The signal intensities of haemorrhage follow a characteristic time course, mainly because of the metabolic processes in and around the haematoma, ${ }^{6813}$ which makes an approximate estimate of the timing of the insult possible. Table 2 shows the grading of the haemorrhages in three stages: (1) acute ( $<3$ days); (2) subacute (4-14 days) and; (3) chronic ( $>2$ weeks). The grading is based on the gradual abnormal signal changes in $\mathrm{T} 1$ and $\mathrm{T} 2$, seen in relation to the timing of the lesion described by previous 
Table 2 Association between age of intracranial haemorrhage and changes in T1- and T2 signals after injury

\begin{tabular}{llll}
\hline & Age of haemorrhage & T1-weighted images & T2-weighted images \\
\hline Acute & $<3$ days & $\begin{array}{l}\text { Iso-to mildly } \\
\text { hyperintense }\end{array}$ & Iso-to hypointense \\
Subacute & $4-14$ days & Hyperintense & $\begin{array}{l}\text { Hypointense centre, hyperintense } \\
\text { periphery } \\
\text { Cyperintense }\end{array}$ \\
\hline
\end{tabular}

investigators. ${ }^{6121321}$ The extent and location of the lesions primarily determine the prognosis. For this reason we grouped the patients according to their MRI findings in (I) diffuse brain injury (here defined as brain lesions that extend beyond a single focal hemispheric lesion), (II) focal hemispheric lesions, and (III) normal.

Head ultrasonography was performed at the bedside in the intensive care unit between days 1-7 (mean 2.5 days) using a $7 / 5.0 \mathrm{mHz}$ transducer through the anterior fontanelle. Ultrasonography and EEG were performed routinely, not as a part of the protocol.

\section{Results}

Based on the MRI and clinical history the causes of seizure activity were $\mathrm{HIE}(35 \%)$; intracranial haemorrhage $(26 \%)$; metabolic disturbances and cerebral dysgenesis (16\%); and unknown (23\%). The clinical diagnosis before the MRI scans was thought to be hypoxic-ischaemic encephalopathy (39\%), metabolic disease (6\%), and unknown (55\%). Infants with diffuse lesions had earlier onset of seizures (mean 1.3 days) than infants with focal lesions (mean 1.9 days). They also had a significantly higher mortality and morbidity (seven died, five had cerebral palsy) in contrast to infants with focal lesions (three with cerebral palsy, six who were normal) (table 3). EEG was abnormal in $83 \%$ of the infants who had diffuse lesions, $89 \%$ of the infants with focal lesions, and in $30 \%$ of the infants with normal MRI. Neurological signs of encephalopathy were most severe in infants with diffuse brain pathology. The Apgar scores and umbilical $\mathrm{pH}$ were the same in all three groups (table 3 ).

To estimate prognosis, the 31 patients were grouped according to the MRI findings, as either diffuse brain injury (brain lesions that extend beyond a single focal hemispheric lesion), focal lesions, and normal. Table 3 summarises the relation between grade of brain lesions and clinical findings.

Table 4 summarises the clinical data of the neonates with seizures and diffuse brain injury. There were 12 infants with diffuse brain injury. On the basis of the scan findings, eight had lesions caused by cerebral hypoxia-ischaemia. In five of these the lesions were acute or subacute (cases $1,2,3,6,7$ ), and in three they were chronic (cases 4,5, and 8). Four infants had severe congenital malformations of the central nervous system (cases 9 to 12) (table 4).

Five infants had clinical evidence of perinatal asphyxia (cases 1 to 5 ). One had experienced severe pre-eclampsia, and another had placental abruption. However, the other three had no apparent cause. Cases 1 and 2 showed only mild MRI changes within the first two days (fig 1). Necropsy findings in case 1, 20 hours after the MRI scan, showed widespread acute

Table 3 The association of brain pathology with different indicators of outcome in 31 neonates with seizures

\begin{tabular}{|c|c|c|c|}
\hline$M R I$ & Diffuse pathology $(n=12)$ & Focal pathology $(n=9)$ & Normal $(n=10)$ \\
\hline Diagnosis & $\begin{array}{l}\mathrm{HIE}(9)^{\star}, \mathrm{CNS} \\
\text { malformations (3) }\end{array}$ & $\begin{array}{l}\text { Subcortical haemorrhage (4), art. cer. media } \\
\text { infarct (2), periventricular haemorrhage (1), } \\
\text { cerebellar haemorrhage (1), dilated left ventricle } \\
\text { (1) }\end{array}$ & \\
\hline Outcome & 7 died, 5 cerebral palsy & 3 cerebral palsy, 6 normal & 10 normal \\
\hline Abnormal EEG & 10 & 8 & 3 \\
\hline Apgar (5 min.) & $2-10,($ median: 9$)$ & 4-10, (median:8) & 1-10, (median:9) \\
\hline Umbilical $\mathrm{pH}$ & 6.73-7.32, (median:7.10) & 7.08-7.32, (median:7.14) & $\begin{array}{l}6.80-7.43 \\
\text { (median:7.05) }\end{array}$ \\
\hline Neonatal encephalopathy & 2-3, (median:3) & $2,($ median: 2$)$ & $1-2,($ median:2) \\
\hline $\begin{array}{l}\text { Abnormal } \\
\text { ultrasonography }\end{array}$ & 2 & 1 & 0 \\
\hline Onset of seizures & 1-2 day, (median: 1 ) & 1-3 day, (median:2) & $\begin{array}{l}\text { 1-3 day } \\
\text { (median: } 1 \text { ) }\end{array}$ \\
\hline
\end{tabular}

$\star=$ for details see table 1 and 4 .

Table 4 Clinical data of neonates with seizures and diffuse brain injury

\begin{tabular}{|c|c|c|c|c|c|c|}
\hline Case No & $M R I$ (day) & Outcome & Perinatal risk signs & $N E$ & Apgar (5 min) & Umbilical pH \\
\hline 1 & $\begin{array}{l}\text { D1: Heterogeneous } \mathrm{T}_{2} \text { SI of } \\
\text { WM }\end{array}$ & $t$ & Pre-eclampsia FB, MF & 3 & 2 & 6.73 \\
\hline 2 & D2: As pt. 1 D7: A + C & $\dagger$ & Placental abruption, MF & 3 & 3 & 6.85 \\
\hline 3 & $\mathrm{D} 2: \mathrm{A}+\mathrm{C}$ & $t$ & $\mathrm{FB}, \mathrm{MA}, \mathrm{MF}$ & 3 & 6 & 6.76 \\
\hline 4 & D27: C & CP & $M F$ & 3 & 3 & 6.90 \\
\hline 5 & D30: $C+E$ & $\mathrm{CP}$ & MF & 3 & 4 & 6.81 \\
\hline $6^{\star}$ & $\mathrm{D} 4: \mathrm{A}+\mathrm{E}+\mathrm{SAH}$ & $\mathrm{CP}$ & $\mathrm{FB}, \mathrm{MSA}$ & 2 & 10 & 7.16 \\
\hline $7^{\star}$ & D2: A + C & $\mathrm{CP}$ & $\mathrm{FB}$ & 3 & 9 & 7.10 \\
\hline $8^{\star}$ & D28: D + C & CP & $\mathrm{FB}, \mathrm{MSA}$ & 2 & 9 & 7.30 \\
\hline $9^{\star}$ & $\mathrm{D} 4: \mathrm{B}+\mathrm{C}$ & $\dagger$ & None & 2 & 10 & 7.19 \\
\hline $10^{\star}$ & D4: CNS malformation & $\dagger$ & Floppy & 2 & 9 & 7.32 \\
\hline $11^{\star}$ & D4: CNS malformation & $t$ & Floppy & 2 & 9 & 7.32 \\
\hline $12^{\star}$ & D7: CNS malformation & $t$ & Microcephalic MSA & 2 & 10 & NA \\
\hline
\end{tabular}

MRI patterns A-E are described in table 1.

$\mathrm{NE}=$ neonatal encephalopathy according to Sarnat ${ }^{17}, \mathrm{D}=$ Day, $\mathrm{SI}=$ signal intensities, WM $=$ white matter, FB $=$ fetal bradycardia ( $<80$ beats $/ \mathrm{min}), \mathrm{MF}=$ multiorgan failure, $\mathrm{MA}=$ meconium aspiration, MSA = meconium stained amniotic fluid, bradycardia $(<80$ beats $/ \mathrm{min}), \mathrm{MF}=$ multiorgan failure, $\mathrm{MA}=$ meconium aspiration, $\mathrm{MSA}=$ meconium stained
$\mathrm{CP}=$ cerebral palsy, $\mathrm{SAH}=$ subarachnoidal haemorrhage, $\mathrm{NA}=$ not available, ${ }^{\star}=$ lesion of antepartum origin. 
Table 5 Clinical data of neonates with seizures and focal brain injury

\begin{tabular}{|c|c|c|c|c|c|c|c|}
\hline $\begin{array}{l}\text { Case } \\
\text { No }\end{array}$ & $M R I$ & Day & Outcome & $\begin{array}{l}\text { Perinatal } \\
\text { risk } \\
\text { signs }\end{array}$ & $N E$ & $\begin{array}{l}\text { Apgar } \\
\text { (5 min) }\end{array}$ & $\begin{array}{l}\text { Umbilical } \\
\text { pH }\end{array}$ \\
\hline 13 & Art. cer. media infarct & 10 & $\mathrm{CP}$ & MSA & 2 & 10 & 7.30 \\
\hline 14 & $\begin{array}{l}\text { Cortical haemorrhage, } \\
\text { nucleus caudatus haemorrhage }\end{array}$ & 8 & $\mathrm{CP}$ & $\begin{array}{l}\text { MSA, } \\
\text { FB }\end{array}$ & 2 & 8 & 7.08 \\
\hline \multirow[t]{2}{*}{15} & $\begin{array}{l}\text { Subdural haemorrhage, } \\
\text { subdural haemorrhage, and }\end{array}$ & 1 & & & & & \\
\hline & periventricular haemorrhage & 7 & Normal & MF & 2 & 4 & 6.86 \\
\hline $16^{\star}$ & Art. cer. media infarct & 9 & $\mathrm{CP}$ & FB & 2 & 4 & 7.24 \\
\hline 17 & $\begin{array}{l}\text { Subcortical haemorrhage and } \\
\text { subarachnoidal haemorrhage }\end{array}$ & 13 & Normal & None & 2 & 7 & 7.08 \\
\hline 18 & $\begin{array}{l}\text { Subcortical haemorrhage and } \\
\text { subdural haemorrhage }\end{array}$ & 6 & Normal & FB & 2 & 7 & NA \\
\hline 19 & $\begin{array}{l}\text { Subcortical haemorrhage, } \\
\text { subdural haemorrhage and } \\
\text { subarachnoidal haemorrhage }\end{array}$ & 3 & Normal & None & 2 & 10 & 7.32 \\
\hline 20 & Cerebellar haemorrhage & 10 & Normal & None & 2 & 10 & NA \\
\hline $21^{\star}$ & Dilated left ventricle & 2 & Normal & None & 2 & 10 & 7.14 \\
\hline
\end{tabular}

$\mathrm{NE}=$ neonatal encephalopathy, $\mathrm{CP}=$ cerebral palsy, $\mathrm{MSA}=$ meconium stained amniotic fluid, $\mathrm{FB}=$ fetal bradycardia $(<80$ beats $/ \mathrm{min}), \mathrm{MF}=$ multiorgan failure, $\mathrm{NA}=$ not available,$\star \star$ lesion of antepartum origin. normal scan (cases 15, 18, 19, 20) and two infants were not allowed to be rescanned.

Clinical follow up ranged from 12-42 months (mean 18.8 months) and showed that one infant, who was extremely hypotonic from birth, was severely mentally and motor retarded (case 16). Two infants had a hemiparesis (cases 13 and 14) and the rest of the infants are normal at the time of writing. None of the infants had focal neurology in the neonatal period.

Ten infants had normal MRI findings. Their first scan was performed on days 1-14 postnatally (mean 7 days). The follow up scan at 3 months of age revealed no brain lesions in the five infants who were allowed to be rescanned. At the age of 12-44 months (mean 32 months) neurodevelopment was normal in all infants. Seizure aetiology was thought to be hypoxicischaemic encephalopathy in the five infants who fulfilled the established criteria, and unknown in the rest.

necrosis. A comparison of the time the scan were performed with the age of the lesions showed that seven infants had lesions of prenatal origin (cases 6 to 12) (table 4). One of them (case 8) had been heavily exposed to heroin; another (case 9) had a molybdenum cofactor deficiency (fig 2). A pair of twins (cases 10 and 11), who were profoundly hypotonic from birth and born of related parents, had defective metabolism of acetyl CoA. Case 12 had a severe malformation of the central nervous system of unknown genesis, and in cases 6 and 7 the aetiology was unknown.

At the three month follow up MRI all infants had delayed myelination. Three also showed thinning of the cortex (cases 4, 7, and 8), and two had cortical (case 6) and subcortical cysts (case 9), together with basal ganglia atrophy. In the short term seven of the 12 infants died, and at the time of writing, one is moderately, and the rest severely, neurodevelopmentally handicapped. Intensive care was withdrawn in two of the infants who died. Nine infants had an intracranial haemorrhage/infarct (table 5).

All lesions were unilateral, with no dominant sided location. The lesions were haemorrhagic, except in one infant (case 13), who had an ischaemic infarct. Two infants were delivered by emergency Caesarean section. One of them had clinical evidence of perinatal asphyxia (case 15), and the other was suspected of having metabolic disease. The mother of case 13 had a large bleeding peripartum. Disseminated intravascular coagulation screening of mother and child was normal. Apart from these three infants, the clinical history gave no clues about a possible cause of the infarcts in the rest of the infants, who were all delivered spontaneously.

Considering the age of the lesions (table 2) at the time of the MRI scan, two of the lesions must have originated some time before delivery. Case 16 had a cystic defect in the left arteria cerebri media region and case 21 had a dilated left ventricle with loss of white matter. The rest of the lesions emerged around birth.

A control scan of the nine infants at 3 months of age showed cystic transformation in two infants (cases 14 and 16) and was unchanged in one (case 21). Four infants had a

\section{Discussion}

MRI in 31 term neonates with seizures indicated a diverse spectrum of brain abnormalities, which emerged at different times owing to different causes. Most brain lesions (61\%) were attributable to hypoxic and or haemodynamic causes. Brain abnormalities were detected by MRI in 21 neonates (68\%). Twelve $(39 \%)$ infants had diffuse brain injury, nine $(29 \%)$ had focal pathology, and $10(32 \%)$ had a normal MRI. The only report on MRI pathology in neonatal seizures by Rollins et $a l^{11}$ which included half as many infants, found the same remarkably high incidence of brain lesions. Diffuse brain injury in neonates with seizures, whatever the aetiology, was associated with a high mortality and morbidity, as $58 \%$ died before the age of 2 years. Among the others, one developed a moderate, and the rest a severe, handicap. In contrast to this there was a considerably better short term prognosis for infants with focal lesions, where one infant had severe cerebral palsy, two moderate cerebral palsy, and the rest were normal at a mean age of 18.8 months. This finding agrees with earlier reports. ${ }^{82}$ None of the infants with a normal MRI had any neurological sequelae at this early stage. The present findings agree with the general consensus, that the prognosis is poor for about half of infants with neonatal seizures. ${ }^{121}$

Besides quantitatively offering a diagnosis in most cases which is imperative for prognosis, MRI also contributed to the understanding of the nature and timing of the brain lesions.

Diffuse brain injury was considered to be prenatal in seven of 12 infants (58\%). Focal lesions originated prenatally in two of nine infants $(22 \%)$. Altogether, neuropathology had an antepartum origin in $43 \%$ of cases. So far no neuroimaging studies, to our knowledge, have tried to document the relative contribution of prenatal events, although there are causal reports of cerebral lesions of fetal onset. ${ }^{23}{ }^{24}$

A model to identify potentially preventable cerebral palsy of intrapartum origin was recently proposed. ${ }^{25}$ However, when the clinical history was reviewed, only one of the lesions 
of intrapartum origin detected here might have been amenable to clinical intervention with the current methods of fetal monitoring.

As in earlier reports, ${ }^{58226-28}$ the aetiology of focal pathology seems more obscure. In spite of a careful review of obstetric and perinatal history no obvious risk factors could be identified except in three of the infants with focal lesions. Intracranial haemorrhage may emerge as the primary cause of neonatal seizures, although more often accompanied by hypoxicischaemic encephalopathy or traumatic injury. ${ }^{5}$ A recent MRI study describing ischaemic and haemorrhagic brain lesions in 14 neonates with seizures ${ }^{28}$ tried to exclude hypoxic-ischaemic encephalopathy as a possible cause of seizures by including only neonates with normal Apgar score and neurology before the onset of seizures. No suggestion of a possible cause was proposed, but $50 \%$ of the infants were delivered by emergency Caesarean section, Kjellands forceps, or ventouse, which may explain some of the lesions. In our study only two of the nine infants with focal lesions were delivered by Caesarean section. Most of the focal lesions were haemorrhagic (89\%), probably indicating an ischaemic pathogenesis due to hypoxia or hypoperfusion perhaps followed by impaired autoregulation and haemorrhage into reperfused ischaemic areas. ${ }^{1529}$ This is supported by the topography of the lesions which show localisation according to the vascular boundary zones in mature neonates- subcortical and periventricular white matter and cerebral cortex in the boundary regions. ${ }^{815} 30$ However, we were unable to show a clear association between focal haemorrhagic lesions and traumatic birth or clinical hypoxia, as only one infant met the criteria for hypoxia. Whether the remaining infants may have had brief periods of hypoxia or hypotension resulting in lesions in the most poorly perfused regions, remains to be determined.

The optimal timing of MRI in acute lesions, where serial investigations are needed, was not the focus of this study. Some of the infants investigated very early on showed clear acute ischaemic lesions. However, two infants investigated within 72 hours had only subtle changes in white matter signal intensities (fig1), when necropsy 20 hours later in one and a rescan five days later in the other revealed extensive ischaemic damage. The value of conventional MRI in the therapeutic decisions made in the first postnatal days needs further elucidation. A recent report found that brain lesions appeared earlier on diffusion weighted imaging than on conventional imaging. ${ }^{31}$

Neonates with seizures and normal MRI had a good short term prognosis, were less neurologically compromised, and generally had a normal EEG. As a group, their Apgar score and umbilical $\mathrm{pH}$ did not differ from that of infants with focal or diffuse brain injury, indicating the poor prognostic value of these variables. ${ }^{3233}$ Although some of these infants had impaired gas exchange in utero as severe as that of infants with focal and diffuse brain lesions, they did not sustain these injuries. This may be explained by the limited period of hypoxia, ${ }^{15}$ or differences in early postnatal care.

Hypoxic-ischaemic encephalopathy is thought to account for two thirds of all causes of neonatal seizures. ${ }^{522}$ Its low incidence as an aetiological factor in the present study may be explained by the additional information received from MRI, a more rigorous definition of the term, or by the small population studied.

The infant with molybdenum cofactor deficiency had brain lesions mimicking hypoxiaischaemia with a possible timing of at least two to three weeks before birth (case 9) (fig 2). It is important to recognise that MRI registers nonspecific morphological changes that may cover different aetiologies, calling for careful clinical research for a reliable diagnosis. There has been some debate about what proportion of neonatal injury is attributable to hypoxicischaemic injury, ${ }^{32}$ and to what extent the signs of postaxphyxial encephalopathy reflect chronic brain lesions of antepartum onset. ${ }^{23242}$ But in the clinical context, MRI is a considerable improvement because it allows us to estimate how many complications really are preventable.

Cranial ultrasonography in term neonates with seizures underestimates the number of brain lesions. $^{781534-36}$ In the present study, ultrasonography identified only $10 \%$ of the lesions seen on MRI, which may partly be explained by the very early scan (mean 2.5 days). The temporal evolution in brain pathology may reveal more abnormalities on subsequent ultrasonography, as evidenced by recent reports. ${ }^{2937}$

An EEG and the duration and degree of neonatal encephalopathy provide very useful information on the severity of the cerebral dysfunction. However, MRI, with its high sensitivity and anatomic resolution, contributes important additional information about the time of occurrence and nature of the neuropathology underlying seizure activity. Mortality and neurological morbidity remain a major risk in one out of two term infants with neonatal seizures. MRI yields significant predictive information which is critical to prognosis.

This study could never have taken place without the support of Professor Ole Henriksen, Danish Research Center of Magnetic Resonance, University Hospital, Hvidovre, Denmark.

We thank the members of the staff of the neonatal unit for qualified assistance with a difficult task.

1 Rose AL, Lombroso CT Neonatal seizure states. A study of clinical, pathological, and electroencephalographic features clinical, pathological, and electroencephalographic features 1970;45:404-25.

Bergman I, Painter MJ, Hirsch RP, Crumrine PK, David R. Outcome in neonates with convulsions treated in an intensive care unit. Ann Neurol 1983;14:642-7.

3 Ellenberg JH, Nelson KB. Cluster of perinatal events identifying infants at high risk for death or disability. $\mathcal{F}$ Pediatr 1988;113:546-52.

4 Scher MS, Aso K, Beggarly ME, Hamid MY, Steppe DA, Painter MJ. Electrographic seizures in preterm and full-term neonates: Clinical correlates, associated brain lesions, and risk for neurologic sequelae. Pediatrics 1993;91:128-34.

5 Hill A, Volpe JJ. Neonatal seizures. In: Roberton NRC, ed. Textbook of Neonatology. 2nd edn. Forlag, 1994: 1043-55.

6 McArdle CB, Richardson CJ, Hayden CK, Nicholas DA Amparo EG Abnormalities of the neonatal brain. MR Amparo EG. Abnormalities of the neonatal brain: MR
imaging. Part II. Hypoxic- ischemic brain injury. Radiology 1987;163:395-403.

7 Keeney SE, Adcock EW, McArdle CB. Prospective observations of loo high-risk neonates by high-field (1.5 Tesla) magnetic resonance imaging of the central nervous 
system,I: Intraventricular and extracerebral lesions. Pediatrics 1991;87:421-30.

8 Keeney SE, Adcock EW, McArdle CB: Prospective observations of 100 high-risk neonates by high-field (1.5 Tesla) magnetic resonance imaging of the central nervous system, II: Lesions associated with hypoxic-ischemic encephalopathy. Pediatrics 1991;87:431-8.

9 Truwit CL, Barkovich AJ, Koch TK, Ferriero DM. Cerebral palsy: MR findings in 40 patients. Am $\mathcal{f}$ Neuroradiol 1992;13:67-78.

10 Barkovich AJ. MR and CT evaluation of profound neonatal and infantile asphyxia. Am f Neuroradiol 1992;13:959-72

11 Rollins NK, Morriss MC, Evans D, Perlman JM. The role of early MR in the evaluation of the term infant with seizures. Am F Neuroradiol 1994;15:239-48.

12 McArdle CB, Richardson CJ, Hayden CK, Nicholas DA Crofford MJ, Amparo EG. Abnormalities of the neonatal brain:MR imaging. Part I.Intracranial hemorrhage. Radibrain:MR imaging. Part
ology 1987;163:387-94.

13 Zuerrer M, Martin E, Boltshauser E. MR imaging of intracranial hemorrhage in neonates and infants at 2.35 Tesla. Neuroradiology 1991;33:223-9.

14 Baenziger O, Martin E, Steinlin M, Good M, Largo R, Burger R, et al. Early pattern recognition in severe perinatal asphyxia: a prospective MRI study. Neuroradiology 1993;35:437-42.

15 Martin E, Barkovich AJ. Magnetic resonance imaging in perinatal asphyxia. Arch Dis Child 1995;72:F62-F70.

16 Volpe JJ. Neonatal seizures: Current concepts and revised classification. Pediatrics 1989;84:422-8.

17 Sarnat HB, Sarnat MS. Neonatal encephalopathy following fetal distress. Arch Neurol 1976;33:696-705.

18 Steinlin M, Dirr R, Martin E, Boesch C, Largo R, Fanconi S, Boltshauser E. MRI following severe perinatal asphyxia: Preliminary experience. Pediatr Neurol 1991;7:164-70.

19 Volpe JJ. Value of MR in definition of neuropathology of cerebral palsy in vivo. Am $\mathcal{F}$ Neuroradiol 1992;13:79-83.

20 Barkovich AJ, Gressens P, Evrard P. Formation, maturation and disorders of brain neocortex. $\mathrm{Am} \mathcal{F}$ Neuroradiol and disorders

21 Gomori JM, Grossman RI, Goldberg HI, Zimmerman RA, Bilaniuk LT. Intracranial hematomas: imaging by highfield MRI. Radiology 1985;157:87-93.

22 Bernes SM, Kaplan AM. Evolution of neonatal seizures. Pediatr Clin North Am 1994;41:1069-104.

23 Scher MS, Belfar H, Martin J, Painter MJ. Destructive brain lesions of presumed fetal onset: antepartum causes of cerebral palsy. Pediatrics 1991;88:898-906.

24 Girard N, Raybaud C, Dercole C, Boubli L, Chau C, Cahen $\mathrm{S}$, et al. In vivo MRI of the fetal brain. Neuroradio 1993;35:431-6.
25 Gaffney G, Flavell V, Johnson A, Squier MV, Sellers S. Model to identify potentially preventable cerebral palsy of intrapartum origin. Arch Dis Child 1995;73:F106-8.

26 Levy SR, Abroms IF, Marshall PC, Rosquete EE. Seizures and cerebral infarction in the full-term newborn. Ann Neurol 1985;17:366-70.

27 Rios A, Goyal M, Kresch MJ, Rao SB, Brion LP. Magnetic resonance imaging in full-term infants with repetitive focal seizures. F Perinatol 1992;XII:252-6.

28 Mercuri E, Cowan F, Rutherford M, Acolet D, Pennock J, Dubowitz L. Ischaemic and haemorrhagic brain lesions in newborns with seizures and normal Apgar scores. Arch Dis Child 1995; 73:F67-74.

29 Hill A, Volpe JJ. Hypoxic-ischaemic cerebral injury. In: Roberton NRC, ed. Textbook of neonatology. 2nd edn. Forlag, 1994:1061-76.

30 Volpe JJ, Herscovitch P, Perlman JM, Kreusser KL, Raichle ME. Positron emission tomography in the asphyxiated term newborn: parasigittal impairment of cerebral blood flow. Ann Neurol 1985;17:287-96.

31 Cowan FM, Pennock JM, Hanrahan KP, Manji KP, Edwards D. Early detection of cerebral infarction and hypoxic ischemic encephalopathy in neonates using diffusion weighted magnetic resonance imaging. Neuropediatrics 1994;25:172-5.

32 Nelson KB, Emery III ES. Birth asphyxia and the neonatal brain: What do we know and when do we know it? Clin Perinatol 1993;20:327-45.

33 Levene MI, Sands C, Grindulis H, Moore JR. Comparison of two methods of predicting outcome in perinatal asphyxia. Lancet 1986;ii:67-9.

34 Babcock DS, Ball WB, Postasphyxial encephalopathy in full term infants: ultrasound diagnosis. Radiology 1983;148:417-23.

35 Siegel MJ, Shackelford GD, Perlman JM, Fulling KH. Hypoxic-ischemic encephalopathy in term infants: diagnosis and prognosis evaluated by ultrasound. Radiology 1984;152:395-9.

36 Eken P, Toet MC, Groenendaal F, De Vries LS. Predictive value of early neuroimaging, pulsed Dopler and neurophysiology in full term infants with hypoxic-ischaemic encephalopathy. Arch Dis Child 1995;73:F75-80.

37 Rutherford MA, Pennock JM, Dubowitz LMS. Cranial ultrasound and magnetic resonance imaging in hypoxicischaemic encephalopathy: a comparison with outcome. Dev Med Child Neurol 1994;36:813-25. 